\title{
TRITIUM - A Quasi Real-Time Low Activity Tritium Monitor for Water
}

\author{
C.D.R. Azevedo, A. Baeza, M. Brás, T. Cámara, C. Cerna, E. Chauveau, J.M. Gil, J.A. Corbacho,V. Delgado, \\ J. Díaz, J. Domange, C. Marquet, M. Macko, M. Martínez-Roig*, A. Moreno, F. Piquemal, A. Rodríguez, \\ J. Rodríguez, C. Roldán, J.F.C.A. Veloso, N. Yahlali \\ * Instituto de Física Corpuscular (IFIC), marcos.martinez@ific.uv.es
}

\begin{abstract}
Tritium is released abundantly to the environment by nuclear power plants (NPP), as a product of neutron capture by hydrogen and deuterium. In normal running conditions, released cooling waters may contain levels of tritium close to or even larger than the maximum authorised limit for human consumption (drinking and irrigation). The European Council Directive 2013/51/Euratom requires a maximum level of tritium in water for human consumption lower than $100 \mathbf{B q} / \mathbf{L}$. Current monitoring of tritium activity in water by liquid scintillating method takes about two days and can only be carried out in a dedicated laboratory. This system is not appropriate for real time monitoring. At present, there exists no available detector device with enough sensitivity to monitor waters for human consumption with high enough sensitivity. The goal of the TRITIUM project is to build a tritium monitor capable to measure tritium activities with detection limit close to $100 \mathrm{~Bq} / \mathbf{L}$, using instrumentation technique developed in recent years for Nuclear and Particle Physics, such as scintillating fibres and silicon photomultipliers (SiPM). In this paper the current status of the TRITIUM project is presented and the results of first prototypes are discussed. A detector system based on scintillating fibers read out either photomultiplier tubes (PMTs) or silicon photomultiplier (SiPM) arrays is under development and will be installed in the vicinity of Almaraz nuclear power plant (Cáceres, Spain) by the fourth term of 2019.
\end{abstract}

\section{INTRODUCTION}

\section{A. The SUDOE TRITIUM Project}

The TRITIUM project is funded by the INTERREGSUDOE program of the European Union and composed by a consortium of 5 southwestern European institutions: University of Extremadura (Spain), University of Valencia (Spain), University of Aveiro (Portugal), CENBG Bordeaux (France) and Junta de Extremadura (Spain). The main aim of the project is to develop and build a monitor for low activity tritium in water with near real-time capability for insitu monitoring of water released by nuclear power plants.

M. Martínez-Roig(corresponding author), T. Cámara, V. Delgado, J. Díaz, and N. Yahlali are with Instituto de Física Corpuscular, Centro mixto CSICUniversidad de Valencia, Paterna, Spain. (email: marcos.martinez@ific.uv.es)

C.D.R. Azevedo, M. Brás and J.F.C.A. Veloso are with I3N - Departamento de Fisica da Universidade de Aveiro, Aveiro, Portugal

C. Roldán is with Universidad de Valencia, Departamento de Física Aplicada, Burjassot, Spain.

A. Baeza, J.A. Corbacho and A. Rodríguez are with Universidad de Extremadura, Laboratorio de Radioactividad Ambiental. Servicio de apoyo a la investigación, Cáceres, Spain

C. Cerna, E. Chauveau, J. Domange, C. Marquet and F. Piquemal are with Université de Bordeaux and CNRS, CENBG, Gradignan Cedex, France.

J.M. Gil, A. Moreno and J. Rodríguez are with Junta de Extremadura, Plaza del Rastro s/n. 06800, Mérida, Spain.

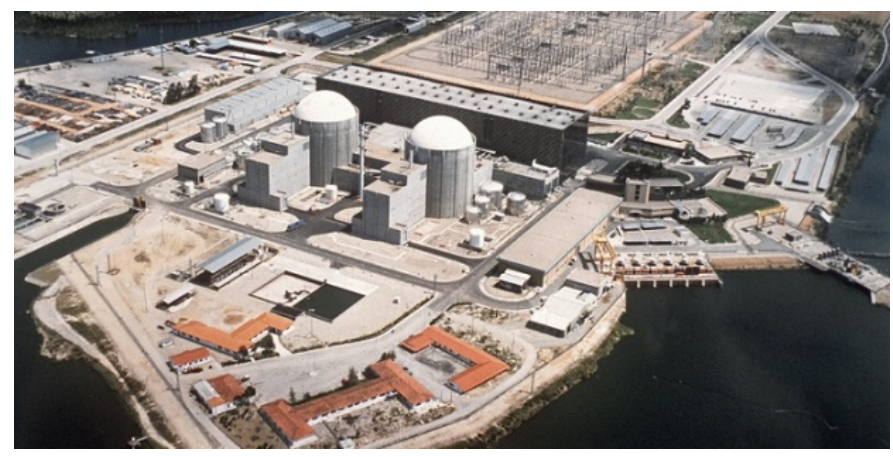

Fig. 1. Picture of Almaraz NPP and Arrocampo dam (Cáceres, Spain).

The EU Council Directive 2013/51/Euratom regulates the maximum level of tritium in water for human consumption to be lower than $100 \mathrm{~Bq} / \mathrm{L}$. This level is considerably higher than that of natural or cosmogenic origin, but may be easily overcome in the cooling water of nuclear power plants even in normal operation. The cooling water is usually released in rivers and may cause direct or indirect contamination of the environment and water for human consumption with tritium, a long-lived hydrogen isotope of more than 12 year halflife. Monitoring the tritium level in the cooling water of nuclear power plants would provide a safety alarm and an indicator of anomalous functioning of the NPP due to an excess of neutrons or a potential water leakage from the primary cooling circuit. Indeed, tritium is the third isotope of hydrogen and is produced by neutron capture of hydrogen and deuterium atoms. Thus, an excess of neutron flux would induce an excess of tritium activity level in the cooling water. In order to measure levels of tritium with the sensitivity required by the European Directive, the consortium is developing a detector system based on scintillating fibers, endowed with active and passive background rejection, both of environmental and cosmic origin. The installation of the detector is planned by the fourth term of 2019, downstream the Arrocampo dam of the Almaraz (Spain) nuclear power plant, in the Tagus river, close to the Portuguese border. A picture of Almaraz NPP is displayed in Fig.1.

\section{B. State of the art}

Measurement of tritium activity is one of the routinely controls to be carried out in the surrounding area of NPPs and, in the future, of nuclear fusion facilities which release tritium 
abundantly. Tritium is an unstable hydrogen isotope with about 12 years half-life, decaying into ${ }^{3} \mathrm{He}$ through $\beta^{-}$decay. The emitted electron has a continuous energy spectrum peaked at $4.7 \mathrm{keV}$ with a maximum energy emission of about $18 \mathrm{keV}$, resulting in an averaged energy of about $6 \mathrm{keV}$. Due to such low energy, the detection of tritium decay is not trivial and requires high sensitivity detectors. There are already available devices for the tritium detection: gaseous detectors, semi-conductors and liquid/solid scintillators. The gaseous detection method consists on the introduction of water vapor into an ionisation chamber; this procedure is quite complex for autonomous operation. Solid state detectors, such APDs, can be directly exposed to the beta radiation but the presence of water makes them unusable. Liquid scintillators have been extensively used in laboratory to detect tritium levels in water as low as $1 \mathrm{~Bq} / \mathrm{L}$, but they require collection and transportation of water samples and data taking during two or more days, which makes them unsuitable for real-time monitoring. Furthermore, they produce highly toxic residues (as toluene), preventing their use for environmental surveillance. Solid scintillators have been employed by different authors: K.J. Hofstetter in 1993 [1] built a capillary system read out by PMTs, obtaining a detection limit of $22000 \mathrm{~Bq} / \mathrm{L}$ in 2 min of measurement. Rathnakaran in year 2000 [2] obtained a detection limit of $10000 \mathrm{~Bq} / \mathrm{L}$ during $10 \mathrm{~min}$ of measurement, a number 2 orders of magnitude higher than the detection limit required in this project. An alternative to solid scintillators, which provides higher sensitivity to low levels of tritium in water, is the employment of scintillating optical fibers as a detection medium. This scintillation alternative, which is proposed in the present work, was investigated already in 1998 by J.W. Berthold and L.A. Jeffers [3], using polystyrene optical fibers with fluor-doped cladding read out by photomultiplier tubes (PMTs). These authors aimed at the development of a detector for monitoring tritium in drinking waters to verify compliance with the US EPA drinking water standard of $740 \mathrm{~Bq} / \mathrm{L}$, a rather larger limit than that required by the present European Directive.

\section{TECHNOLOGICAL ASPECTS OF THE TRITIUM DETECTOR}

The design of TRITIUM detector is based on uncladded scintillating fibers with fluor-doped core, in order to maximize the detection area exposed to water. This is necessary because of the small energy ( $6 \mathrm{keV}$ on average) of electrons from tritium decay to be measured, which prevent the use of cladded comercial fibers, which clad is too thick to be crossed by the tritium decay electrons. Scintillating fibers of $1 \mathrm{~mm}$ and $2 \mathrm{~mm}$ diameter have been considered in the $\mathrm{R} \& \mathrm{D}$ phase, while for light detection the options in study are PMTs or silicon photomultiplier arrays(SiPMs). The latter have the advantage of low polarisation voltages $(\sim 50 \mathrm{~V}$ instead of typically $\sim 1 \mathrm{kV}$ needed by PMTs), robustness and easy temperature dependence correction that may be implemented automatically. On the other hand, using PMTs has the advantage of a lower number of electronic channels and a larger detection area. In both options, the photodetectors reading out a fiber bundle

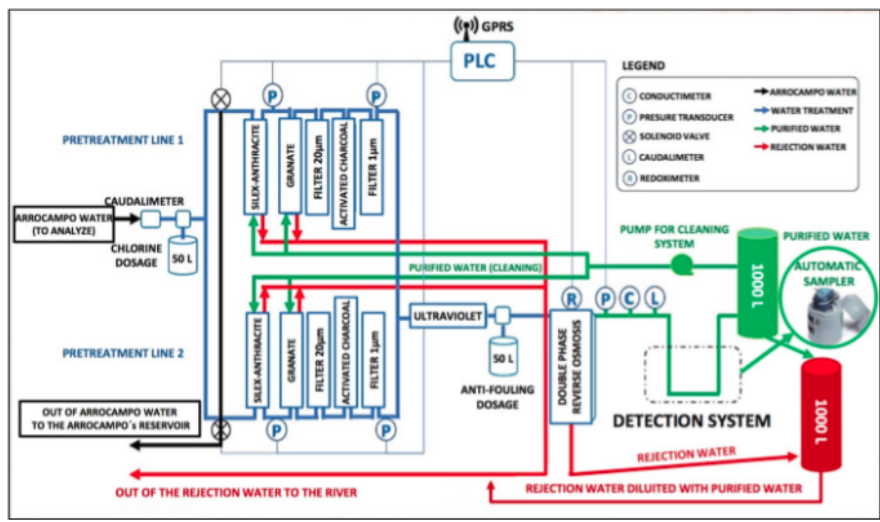

Fig. 2. Scheme of the TRITIUM water purification system delivering ultra pure water to the TRITIUM monitor.

work in coincidence mode in order to reject intrinsic noise (or self-emission in the case of PMTs), and in anti-coincidence mode with a cosmic ray discrimination system made of plastic scintillators. The water supplied to the detector, has to undergo a thorough purification process in order to avoid maintenance of the detector due to organic and mineral depositions on the fiber surface and on the water-vessel inner surfaces. A water purification system was developed to provide a level of water purity specified as hyperpure, characterized by a conductivity of $10 \mu \mathrm{S} / \mathrm{cm}$. In addition, the purification system has to preserve the in-water tritium level prior to the purification process, and ensure an adequate water flow in the detector, allowing the renewal of its water content every $10 \mathrm{~min}$. The detector system has to be able of providing an alarm signal in case a tritium level in water above $100 \mathrm{~Bq} / \mathrm{L}$, after a continuous measurement of about 10 min duration, is found. A scheme of the purification system is displayed in Fig. 2

\section{Detector Development}

\section{A. Simulations of the scintillation process in the fibers}

The proposed detector design using scintillating fibers of $1 \mathrm{~mm}$ or $2 \mathrm{~mm}$ diameter has been extensively studied through GEANT4 simulations [4]. Due to the low energy of the electrons emitted by tritium, and considering that the measurement medium is water, only a small part of the electrons originated by tritium decay close to the fiber surface are detected, because those electrons produced far away loose their energy in the water. The relationship between the energy deposited by electrons in the fiber and the distance from their emission point to the fiber surface was studied by simulating $2 \mathrm{~mm}$ and $1 \mathrm{~mm}$ fibers inside a water cylinder of $0.5 \mathrm{~mm}$ thickness, containing tritium uniformly distributed in the water volume. The electron emission was considered to be spatially isotropic and its energy was sampled from the tritium emission spectrum (Fig. 3, green distribution). The results are shown in Fig. 4, where it is observed that most $(99.7 \%)$ of the events reaching the fiber surface are emitted at a distance less than $5 \mu \mathrm{m}$ from it. Therefore, only are detected those decays happening in a very thin layer of water in the vicinity of the fiber surface. For this reason, scintillating optical fibers cannot be cladded, as the thickness of commercial clads exceed largely $5 \mu \mathrm{m}$. 


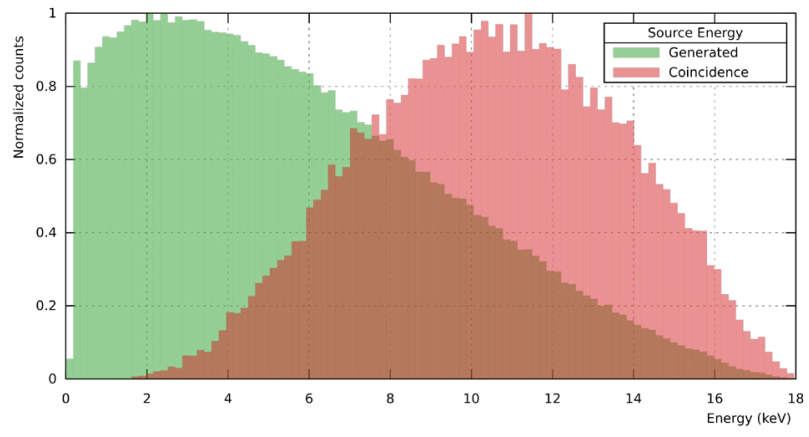

Fig. 3. Energy distribution of electrons from tritium decay (green) and their energy deposited in a scintillation fiber of $2 \mathrm{~mm}$ diameter (red) immersed in tritiated water.

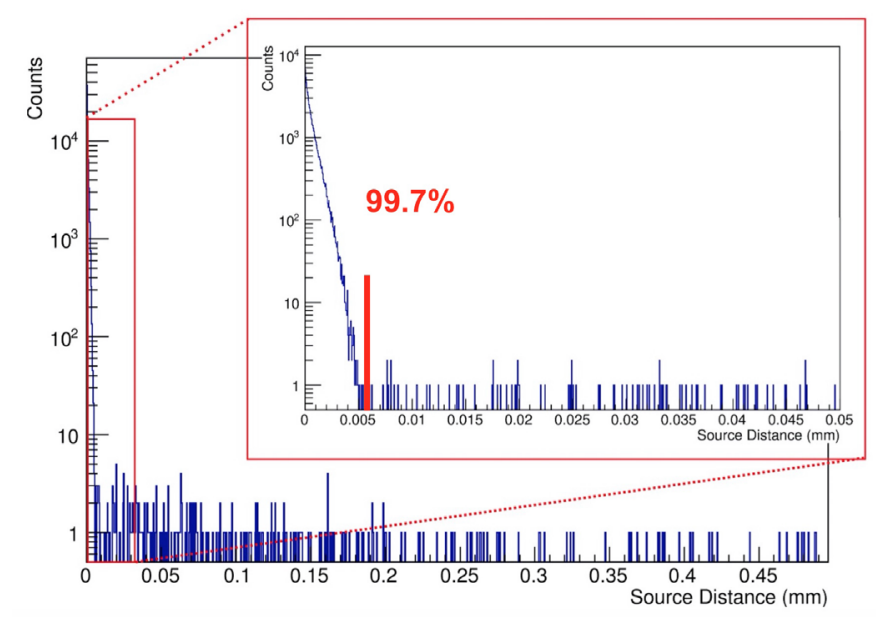

Fig. 4. Distribution of $\beta^{-}$electrons hitting a scintillation fiber immersed in tritiated water as a function of the distance between the beta source and the fiber surface. $99.7 \%$ of the events reaching the fiber surface are produced at a distance less then $5 \mu \mathrm{m}$ from it.

The energy distribution of electrons that reach the fiber surface was studied. The plots displaying the results obtained arepresented in Fig. 3, where, in green, is the tritium emission spectrum, and, in red, the energy distribution of the electrons reaching the fiber, normalized to the emission spectrum in order to be easily compared. The shift of the energy peak of the electrons in the fiber, indicates, that the less energetic electrons can not reach the fibers, as they loose their energy in the water. It must be pointed out that the spectrum of energy deposition in the fiber shows those electrons reaching the fiber surface, which does not necessarily imply that they produce enough photons to be detected.

A crucial issue of the detector design is the diameter of the scintillating optical fibers to be employed. This is determined by a compromise between the detection efficiency of the fibers determined from their light yield collection per unit of energy deposited, and the amount of light produced by energy deposited from the background (cosmic rays and environmental radioactivity essentially), which should be as low as possible. In the actual monitor, a lead shielding will be employed, that should stop most of the environmental radioactive background and soft cosmic rays (essentially electrons and positrons).

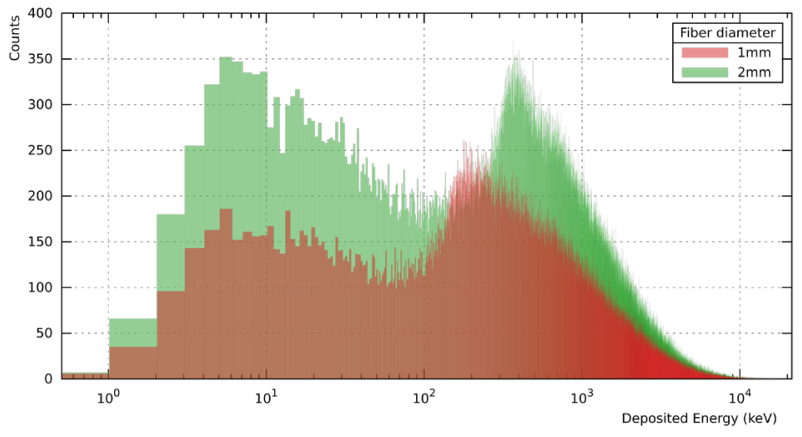

Fig. 5. Distribution of cosmic muon events as a function of the deposited energy in fibers of $1 \mathrm{~mm}$ (red) and $2 \mathrm{~mm}$ (green) diameter.

Therefore, we have studied the hard component of the cosmic ray spectrum, composed mostly at sea level by muons of energy larger than $200 \mathrm{MeV}$. In Fig. 5, the energy deposited by cosmic muons in fibers of $1 \mathrm{~mm}$ and $2 \mathrm{~mm}$ diameter (red and green spectra respectively) are compared. As may have been expected, the counting rate of cosmic muons in a fiber of $1 \mathrm{~mm}$ diameter is smaller than in a fiber of $2 \mathrm{~mm}$ diameter in the whole energy range, and specially in the region of interest for tritium detection $(<8 \mathrm{keV})$. Hence, scintillating fibers of $1 \mathrm{~mm}$ diameter favour background rejection, increasing the detector sensitivity to low tritium activity. Furthermore, it is essential to reduce strongly the cosmic and natural radioactivity backgrounds in the detector. A cosmic ray VETO detector, composed of $1 \mathrm{~cm}$ thick plastic scintillators placed both below and above the TRITIUM monitor, read out by photosensors, has been designed. The whole detection system is enclosed inside a lead castle of several $\mathrm{cm}$ thickness that will, in addition, reduce cosmic muons and natural radioactivity.

\section{B. Prototypes}

Several TRITIUM prototypes were built for the detector proof of concept and efficiency, light yield and sensitivity studies. The first prototype, called TRITIUM-0, contained a bundle of 35 scintillating optical fibers of $1 \mathrm{~mm}$ diameter, without cladding, from Saint-Gobain Crystals [5], terminated in the ends by the connectors exhibited in Fig. 6. The fiberbundle was introduced into a U-shaped PVC tube, that allowed vertical coupling of the fibers to two PMTs, as depicted in Fig. 7. Optical grease BC630 from Saint-Gobain Crystals was used to optimise the light transmission to the photosensors. This prototype was first filled with $40 \mathrm{~mL}$ of hyperpure water for background measurements, and afterwards, it was filled with hyperpure highly tritiated water $(108.11 \mathrm{MBq} / \mathrm{L})$ for tritium activity measurement. This prototype proved the feasibility of the detection principle with scintillating fibers, but also showed important issues related to the light production and collection in the detector.

Two additional prototypes, called TRITIUM-1, were built at IFIC and Aveiro for light collection studies, long-term stability studies of scintillating fibers in water, readout and mechanical studies, including implementation of water flow solutions inside the vessel, water-tightness and optical coupling solutions for the photosensors (PMTs or SiPM arrays). 


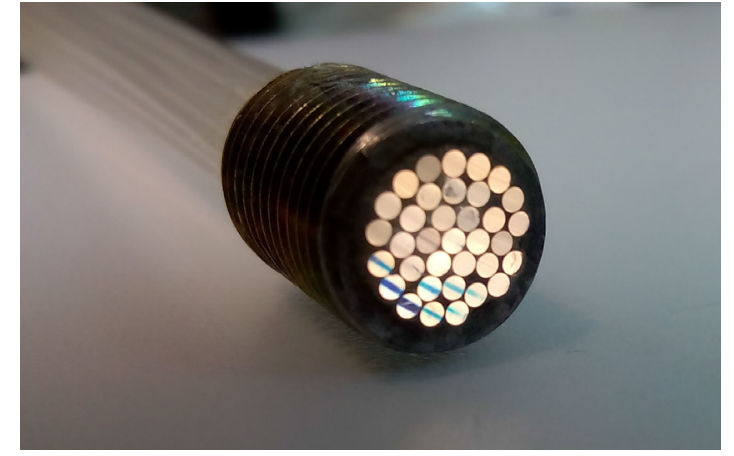

Fig. 6. One of the two end-connectors of the fiber-bundle of TRITIUM-0 prototype. The fiber ends are polished and coupled to the PMT with optical grease BC600 from Saint-Gobain Crystals [5].

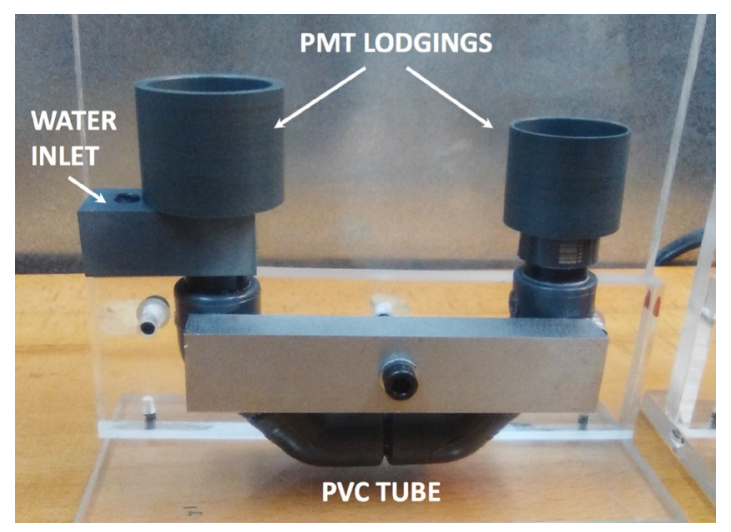

Fig. 7. Proof-of-principle prototype TRITIUM-0 instrumented with two PMTs. The fiber-bundle in the PVC tube was composed of 35 scintillating fibers BCF-12 of $1 \mathrm{~mm}$ diameter, $20 \mathrm{~cm}$ long and without cladding, from Saint-Gobain Crystals.

IFIC prototype was composed of 64 scintillating fibers BCF12 from Saint-Gobain Crystals, of $1 \mathrm{~mm}$ diameter without cladding, lodged in a highly reflective PTFE vessel. A protocol for conditioning of fiber surface was developed to optimize their in-water detection and light collection capability. This includes cleaving, polishing, and surface conditioning in a clean room, which ensures optimal cleanliness and wetting conditions (optimal contact with water) of the fibers surface. The fibers were then introduced into two PTFE matrices, as shown in Fig. 8, that allowed a separation of $1 \mathrm{~mm}$ between neighbour fibers and maintained them in vertical position in the PTFE vessel, as shown in Fig. 9. On top of the vessel, a Hamamatsu R8520-406 PMT was lodged and put into contact with the fibers using optical grease. The PMT signal was preamplified, amplified and acquired in an AmpteK MCA8000D digital Multichannel Analyzer.

A second version of TRITIUM-1, built in Aveiro, used also a PTFE vessel within which a high number (400) of tightly packed $2 \mathrm{~mm}$ diameterscintillating fibers was introduced. These were read out in coincidence mode by two Hamamatsu R2154-02 PMTs, through transparent PMMA windows of the water-vessel. A drawing of this prototype is shown in Fig. 10.

A third monitor TRITIUM-2 was developed for the final monitor to be installed in Arrocampo dam. This monitor makes use of the Aveiro TRITIUM-1 monitor design, and contains

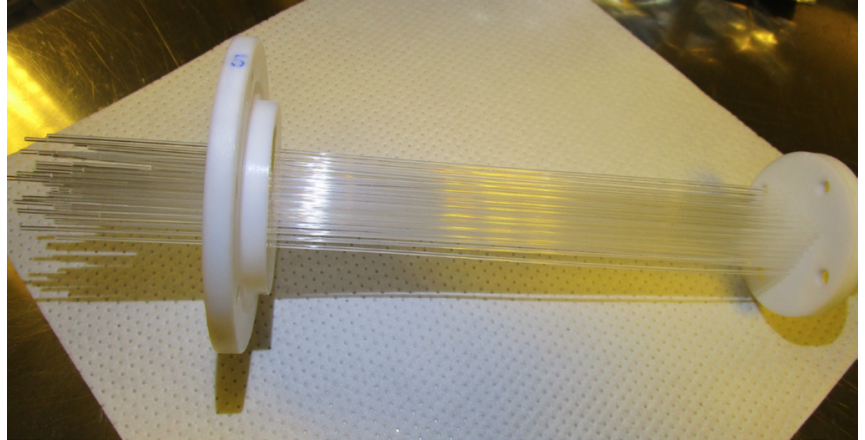

Fig. 8. Scintillating fibers mounted into two PTFE matrices used as top and bottom covers of TRITIUM-1 water vessel. The fibers end were polished and their surface conditioned in a clean room.

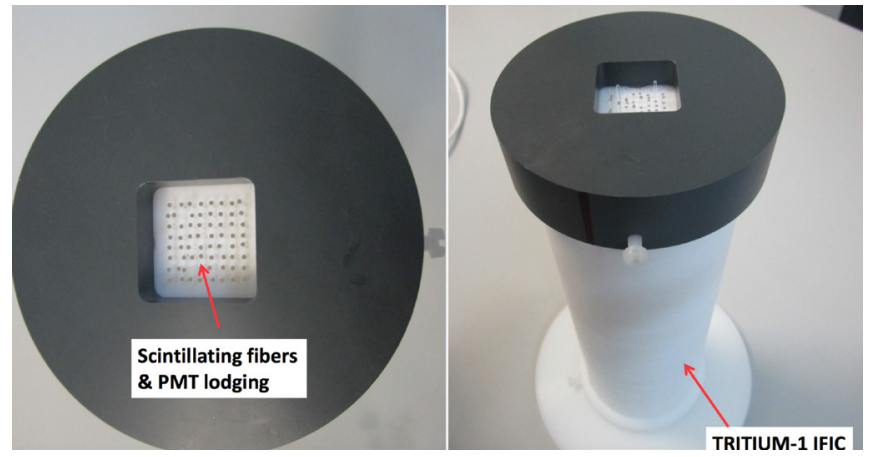

Fig. 9. TRITIUM-1 IFIC prototype with 64 scintillating fibers lodged vertically in a PTFE vessel (right picture), on top of which a PMT was placed in contact with the fibers. The prototype was placed in a dark chamber for data taking.

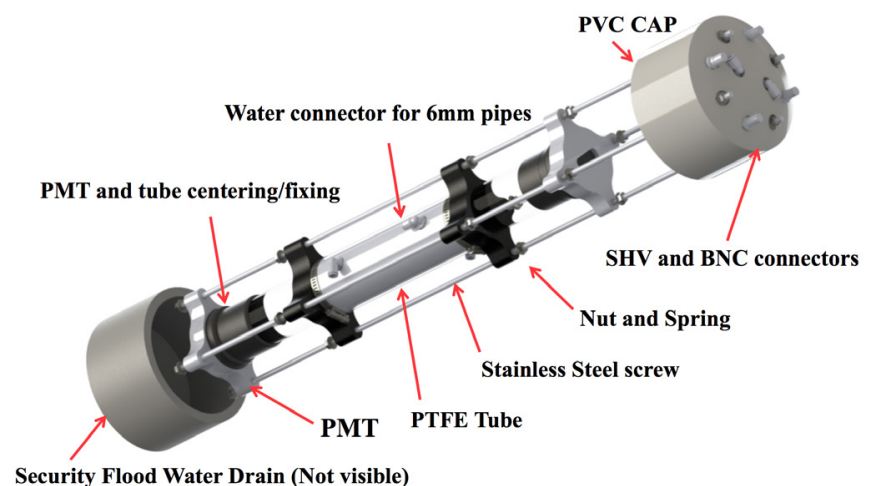

Fig. 10. TRITIUM-1 Aveiro prototype with 400 scintillating fibers, read out by two PMTs in coincidence mode. The fibers are lodged into a PTFE vessel closed by two PMMA windows which transmit the scintillation light to the PMTs. An inlet and an outlet for water flow are foreseen in the vessel body.

$5001 \mathrm{~mm}$ closely packed scintillating fibers, contained in a parallelepiped container with teflon walls to optimise light reflection. This prototype is read-out by SiPM arrays. The details of this prototype are shown in Fig 11

\section{FINAL MONITOR}

A final monitor composed of 15 TRITIUM-2 modules will be installed in Arrocampo dam during the third term of 2019. The system is conceived as composed of an arbitrary number 


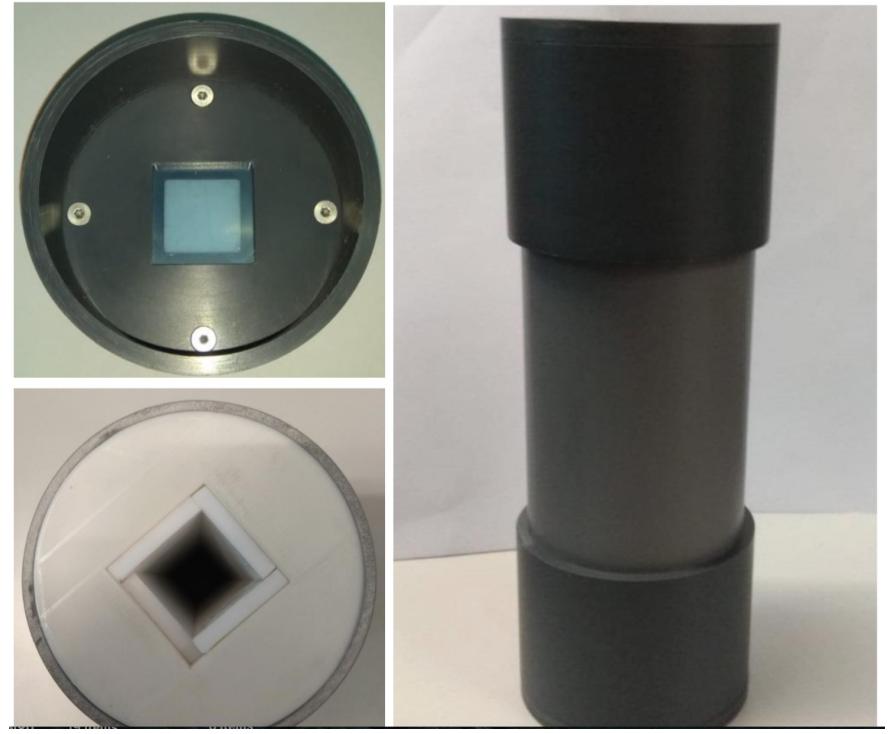

Fig. 11. TRITIUM-2 prototype with 500 scintillating fibers, read out by two photosensors in coincidence mode. The fibers a re 1 odged in a P VC vessel, shown on the right part of the picture, within a parallelepiped container with teflon walls, shown in the picture, to o ptimise light reflection. An inlet and an outlet for water flow w ill be p laced in the v essel b ody to a llow for water circulation. The prototype is read in coincidence mode by photosensors, through PMMA windows shown on the left part of the picture.

of modules, which allows to improve the sensitivity by just increasing the number of modules. The modules will be placed within the metallic structure holding the lead shield shown in Fig 12 . The modules will be read by $4 \times 4$ Hamamatsu SiPM arrays. Data will be acquired by a PETSYS system, developed by PETsys Electronics for PET data acquisition. This system has the capability of reading thousands of SiPM channels with good energy and time resolution and the possibility of making custom triggers. The active cosmic veto is made of two $1 \mathrm{~cm}$ layers of plastic scintillator povided by EPIC Crystals. Two different veto configuration a re b eing s tudied at p resent: two single layers covering the whole set of modules, and partial vetos for groups of three modules as shown in Fig. 14. This latter configuration $\mathrm{h}$ as $\mathrm{m}$ echanical a nd $\mathrm{c}$ abling advantages, and the possibility of putting individual plastics into different trigger configurations a nd i mproving the p osition dependent response. The whole system is depicted in Fig.15.

To verify the lead shielding capability to reject background, an Aveiro module shown in Fig. 8, was installed in Arrocampo dam monitoring station, and measurements were carried out without shielding, with a shielding of $0.5 \mathrm{~cm}$ and with a shielding of $1 \mathrm{~cm}$. The count rates obtained are shown in Fig. 16 was installed in the Arrocampo dam. Measurements were carried out without shielding, with a lead shield $2 \mathrm{~mm}$ thick, and a lead shield $1 \mathrm{~cm}$ thick. The count rate measured is shown in Fig. 17. Curve A corresponds to the measurements carried out without shielding. Curve B corresponds to the measurements acquired with the $2 \mathrm{~mm}$ thick shielding and curve $\mathrm{C}$ to the $1 \mathrm{~cm}$ thick shielding. It is seen from the plots that the shielding reduce strongly the count rate due to low energy background, composed essentially by environmental radioactivity and soft cosmic rays.

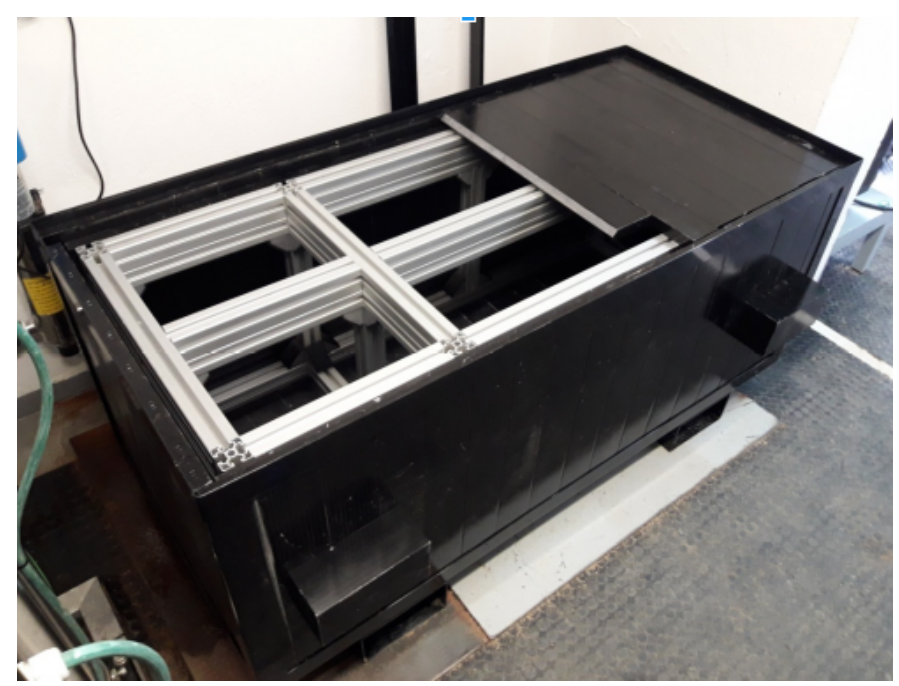

Fig. 12. Metallic structure holding the lead shied in which the TRITIUM-2 modules will be placed.

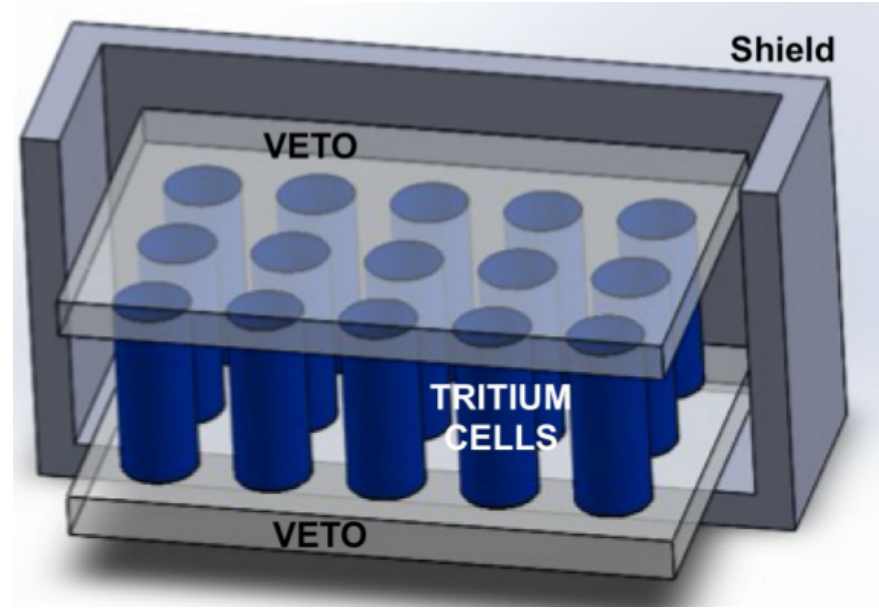

Fig. 13. Scheme of how the TRITIUM-2 modules will be placed within the metallic structure holding the lead shield.

\section{Preliminary Experimental Results}

A clear signal of tritium was observed in the proofof-concept prototype TRITIUM-0 as shown in Fig. 18, in which the signal from tritiated hyperpure water is observed (blue spectrum) above the background signal (red spectrum) from a similar volume of hyperpure water without tritium. However, the signal observed from highly tritiated water (108.11 MBq/L) was weak, clearly indicating poor light production, collection or transmission to the PMTs. Indeed, among the issues identified to determine the tritium signal magnitude are the geometrical configuration of the fiberbundle, the condition of the fibers surface, the water circulation pattern in the detector vessel, the reflectivity of the inner surfaces of the water-vessel, and, of crucial importance for low activities, the magnitude of the background signal in the detector.

In TRITIUM-1 prototypes the fibers underwent a thorough conditioning protocol and were set up straight, avoiding light losses due to bending and poor contact with water. In Fig. 19, 


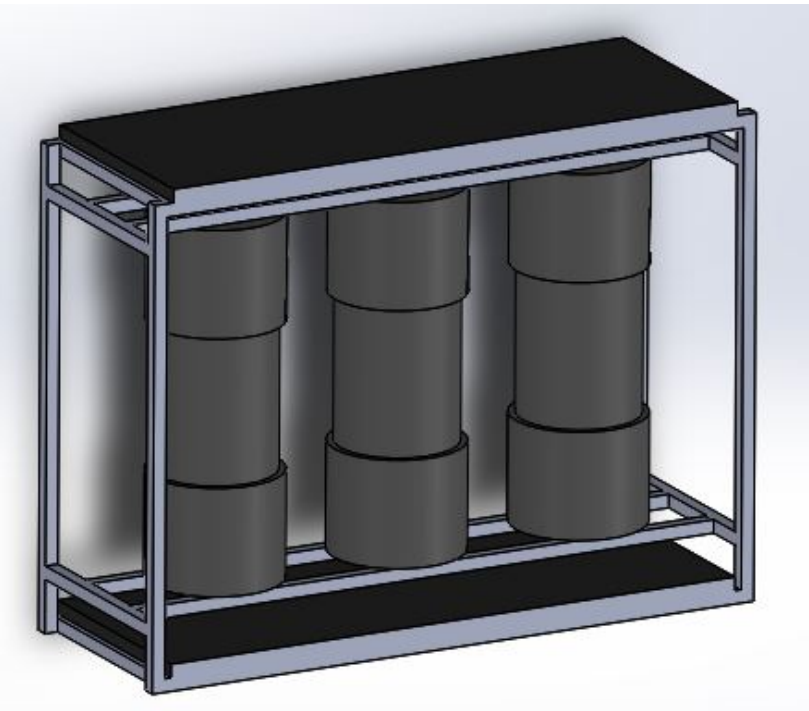

Fig. 14. Scheme of plastic VETO for three TRITIUM-2 modules will be placed within the metallic structure holding the lead shield.

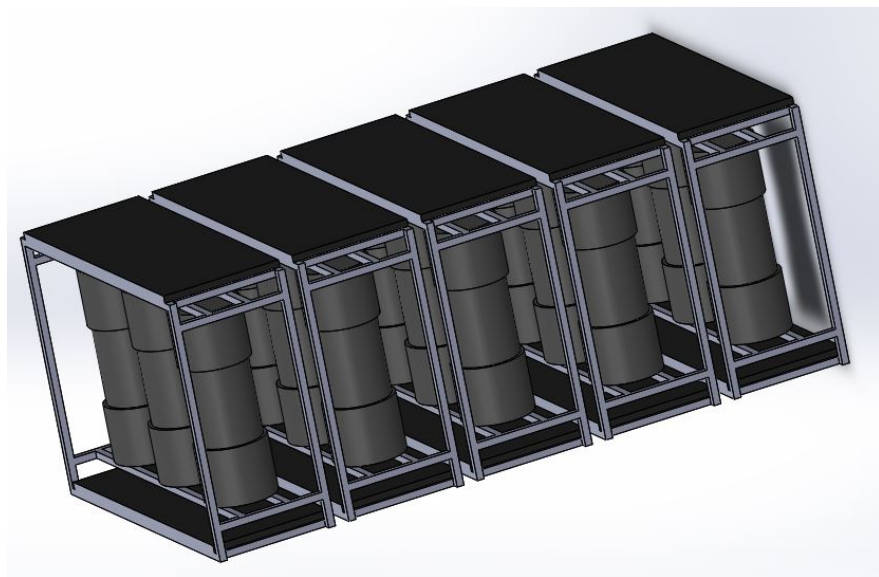

Fig. 15. Scheme of scintillating plastic VETO for 15 TRITIUM-2 modules placed within the metallic structure holding the lead shield.

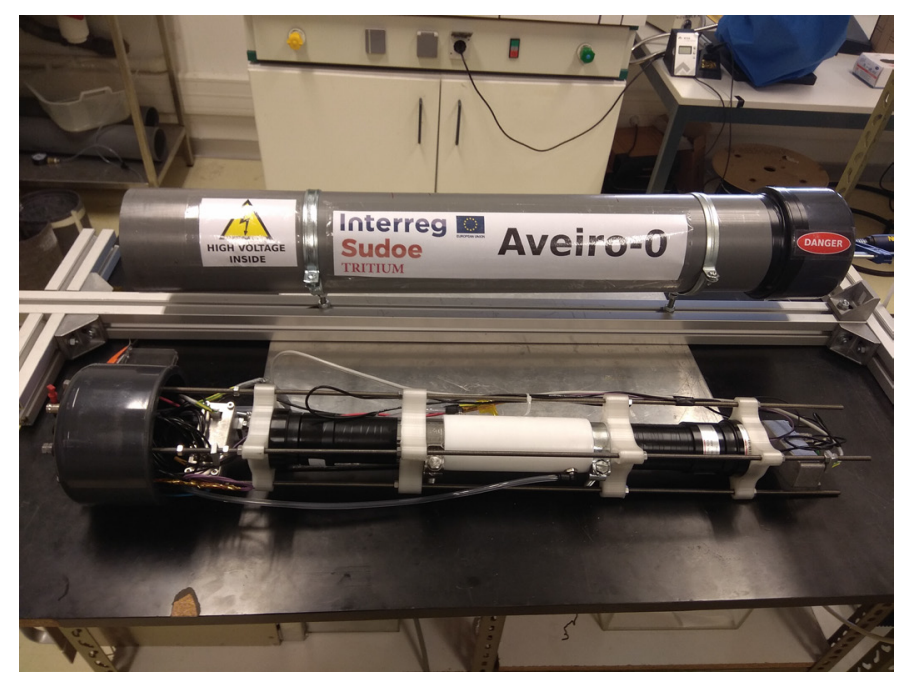

Fig. 16. Aveiro TRITIUM prototype installed in Arrocampo damm

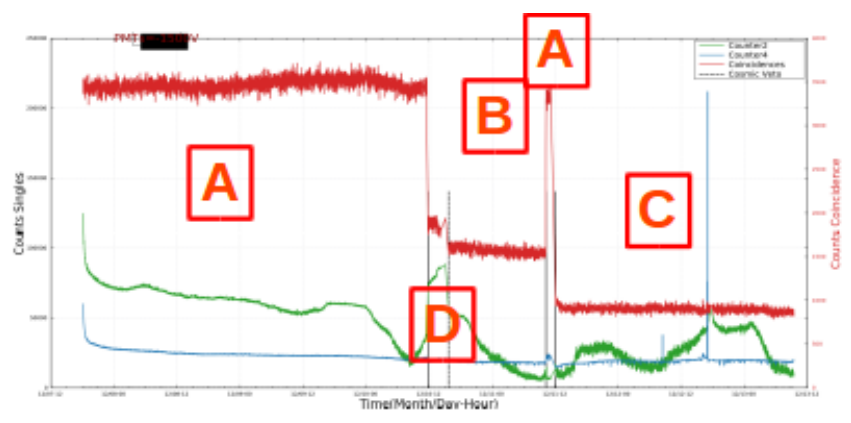

Fig. 17. Results of measurements of the Aveiro TRITIUM prototype installed in Arrocampo damm with different shield conditions.

the signal from tritiated water with similar activity as for TRITIUM- 0 , shows an improvement of the tritium signal by one order of magnitude compared to TRITIUM-0. TRITIUM1 IFIC was also used to assess the stability in water of fiber response over time. Through regular measurements in the laboratory, the detector prototype proved a stable response over a period of 9 months. Further measurements are foreseen in the lead shield in Almaraz TRITIUM site, to assess its increase of sensitivity associated to background rejection.

In TRITIUM-1 Aveiro prototype, a substantial increase in tritium level sensitivity compared to the IFIC-0 and IFIC-1 prototypes is expected, due to the higher number of fibers, producing a larger total detection surface, and also, due to the reduction of the PMTs noise through the implementation of a coincidence readout of the fibers. Before the use of tritiated water in this prototype, the capability of low energy detection by the scintillating fibers was first tested. For that, a small bundle of 12 Saint-Gobain BCF-12 fibers without cladding were assembled and wrapped in teflon tape. The fibers were positioned between 2 Hamamatsu R2154-02 PMTs acquiring in a coincidence window of $500 \mathrm{~ns}$ through a CAEN V1724 digitizer. The PMTs signals where preamplified by a Cremat Cr-110 module and the fibers where irradiated with $5.9 \mathrm{keV}$ photons provided by a ${ }^{55} \mathrm{Fe}$ source. A study of the number of detected events as a function of the PMTs voltage was carried out and the results are presented in the insert of Fig. 20. The increase of the number of events observed with voltage increase in the PMTs is due to the increase of the PMTs detection efficiency, until a plateau is reached at around $1300 \mathrm{~V}$. In the main plot of Fig. 20 the pulse-height amplitude for both PMTs and the corresponding distribution of their sum can be observed. The different distributions for the PMTs are due to the difference in the intrinsic gain although they were biased with the same voltage $(-1250 \mathrm{~V})$. The prototype installed in Arrocampo damm monitoring station shows good stability over time, as shown in Fig. 21, where the count rate over a period of 2 months is displayed. The peaks in the plot correspond to openings of the shielding for maintenance operations. These peaks put in evidence the important role played by the lead shielding. 


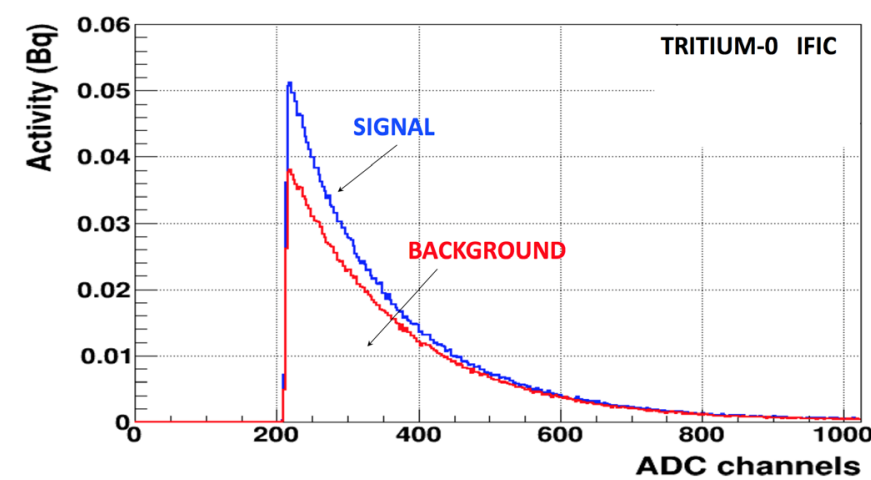

Fig. 18. Data spectra of hyperpure water (red) and of hyperpure tritiated water (108.11 MBq/L, blue) in number of ADC channels, from TRITIUM-0 prototype.

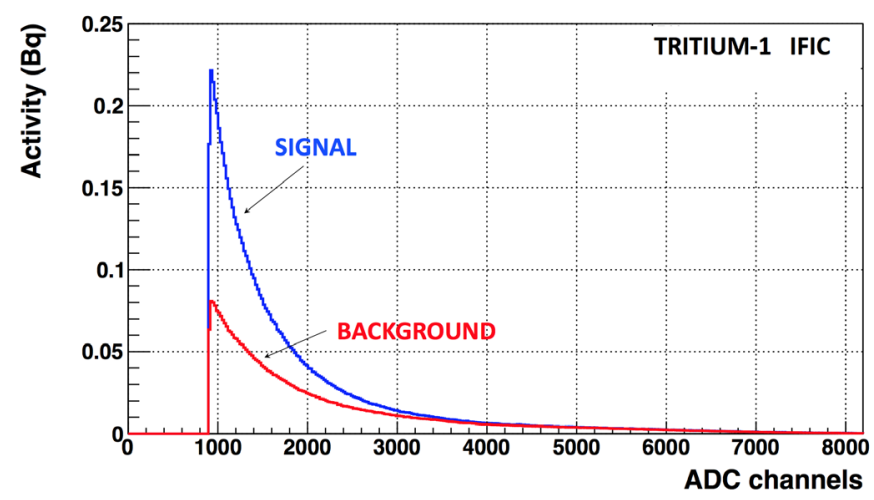

Fig. 19. Data spectra of hyperpure water (red) and of hyperpure tritiated water (108.11 MBq/L, blue) in number of ADC channels, from TRITIUM-1 IFIC prototype.

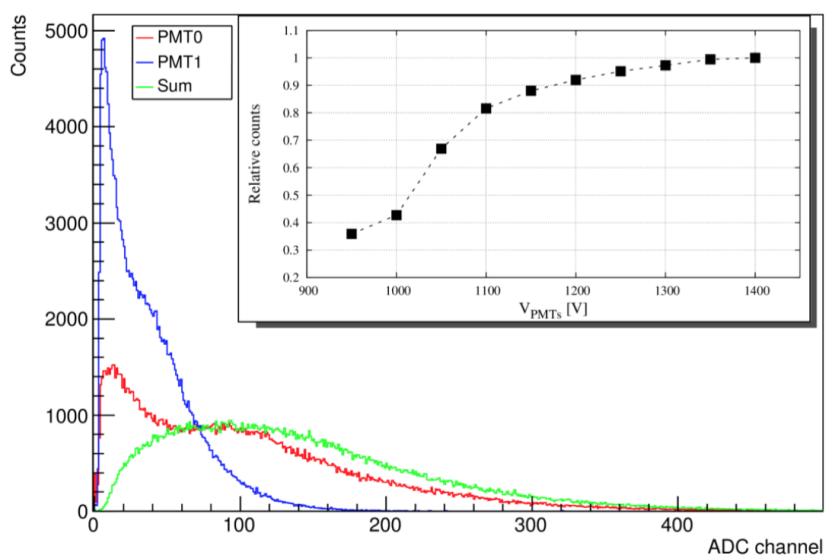

Fig. 20. Pulse-height distribution for each of the PMTs $(V=-1250 \mathrm{~V})$ of TRITIUM-1 Aveiro, and their sum. In the insert is the normalised count rate as a function of PMTs voltage when operating in coincidence mode. The dashed line is just a guide-to-the-eyes.

\section{CONCLUSION AND OUTLOOK}

The TRITIUM project aims at the design, construction and commissioning of a near real-time automatic detector system for monitoring low levels of tritium in water released by nuclear power plants. The tritium monitor has to measure tritium

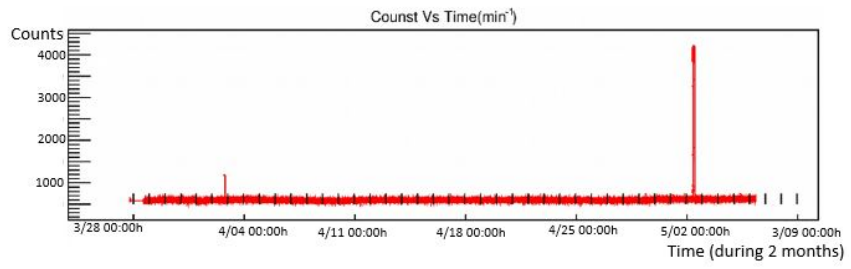

Fig. 21. Count rate of TRITIUM-1 Aveiro prototype during a period of 2 month. The peaks in the plot correspond to openings of the shielding for maintenance operations.

levels below $100 \mathrm{~Bq} / \mathrm{L}$, according to the limit required by the European Council Directive 2013/51/EURATOM for water for human consumption. The final design of the TRITIUM detector employs scintillating optical fibers read out by silicon photomultiplier arrays.

GEANT4 simulations were carried out to optimize the detector design (scintillating fiber length, diameter, geometrical configuration) and several prototypes were developed and operated in the laboratories of the consortium, with the aim of providing a proof of concept of the detector, and for studying its sensitivity and mechanical configuration stability. The simulations indicate that the deposited energy in fibers of $2 \mathrm{~mm}$ diameter without cladding are peaked around $12 \mathrm{keV}$, with a distribution ranging from 2 to $18 \mathrm{keV}$. Moreover the electrons that reach the fiber surface are created at a maximum distance of $5 \mu \mathrm{m}$ from the fiber surface. The possibility to use scintillating fibers to detect, in coincidence mode, low energies such as the $5.9 \mathrm{keV}$ photons from a ${ }^{55} \mathrm{Fe}$ source, was demonstrated as well as the scaling of the detector sensitivity with the increase of the number of scintillating fibers and the optimization of their surface conditions and geometrical configuration.

The results of this $R \& D$ studies allowed the design of a modular TRITIUM detector, composed of 15 cylindrical cells containing each, 500 scintillating optical fibers of $25 \mathrm{~cm}$ long, $1 \mathrm{~mm}$ diameter, without cladding. Each cell will be instrumented with two SiPM arrays which read out the scintillation signals in coincidence mode to reject the intrinsic noise of the photodetectors. Additionally, active and passive shielding based on scintillating plastics and lead bricks respectively, will be used for the rejection of cosmic and natural radioactivity backgrounds in the tritium detector. This shielding is essential to reach high sensitivity to tritium levels below $100 \mathrm{~Bq} / \mathrm{L}$. A water purification system was developed to supply the detector with hyperpure water with conductivity close to $10 \mu \mathrm{S} / \mathrm{cm}$. This system which has been shown to preserve the tritium level existing prior to the purification process, provides a water flow that enables renewal of the detector water-content each $10 \mathrm{~min}$ for tritium measurement.

The construction of the full TRITIUM system is currently in progress. Its commissioning is foreseen in the fourth term of 2019 in the monitoring station of Arrocampo dam at Almaraz (Spain) nuclear power plant. 


\section{ACKNOWLEDGMENT}

This work was supported by the INTERREG-SUDOE EEC program through the project TRITIUM - SOE1/P4/E0214

\section{REFERENCES}

[1] K. J. Hofstetter, Continuous monitoring for tritium in aqueous effluents at SRS using solid scintillators, Trans. Am. Nucl. Soc. (United States), 1993.

[2] M. Rathnakaran et al., A real-time tritium-in-water monitor for measurement of heavy water leak to the secondary coolant 2000 .

[3] J.W. Berthold, and L.A. Jeffers. IN-SITU TRITIUM BETA DETECTOR. United States: N. p., 1998. Web. doi:10.2172/836625.

[4] GEANT4: http://geant4.cern.ch/.

[5] https://www.crystals.saint-gobain.com/. 